\title{
Frequency, location, and association with dental pathology of mucous retention cysts in the maxillary sinus. A radiographic study using cone beam computed tomography (CBCT)
}

\author{
Andy Wai Kan Yeung ${ }^{1} \cdot$ Ray Tanaka $^{1} \cdot$ Pek-Lan Khong $^{2} \cdot$ Thomas von Arx $^{3}$. \\ Michael M. Bornstein ${ }^{1}$
}

Received: 26 June 2017 / Accepted: 7 September 2017 /Published online: 17 September 2017

(C) Springer-Verlag GmbH Germany 2017

\begin{abstract}
Objectives The purpose of the present study was to evaluate the frequency, locations, and dimensions of mucous retention cysts of the maxillary sinus and analyze potential associated dental pathology.

Materials and methods A total of 156 cone beam computed tomography $(\mathrm{CBCT})$ scans were included in the analysis, resulting in an evaluation of 310 maxillary sinuses. The presence of mucous retention cysts (MRC) manifesting as dome-shaped radiopacities in the sinus was diagnosed. Their locations were recorded, and dimensions $(\mathrm{mm})$ were measured in coronal and sagittal/axial slices. The patients were grouped into (a) patients/sinuses with MRCs (test), and (b) patients/sinuses with healthy or any other changes (control) for further comparison and evaluation.

Results There were 40 sinuses (12.9\%) with a presence of a total of 56 MRCs. The mean age of involved patients was 29.0 years. The analysis showed that gender, age, sinus side, status of dentition, endodontic status, and periodontal status did not have a significant influence on the presence of MRCs when compared between test and control groups. Age and endodontic status exhibited a significant association with cyst location.
\end{abstract}

Michael M. Bornstein

bornst@hku.hk

1 Oral and Maxillofacial Radiology, Applied Oral Sciences, Faculty of Dentistry, The University of Hong Kong, Hong Kong, SAR, China

2 Department of Diagnostic Radiology, Li Ka Shing Faculty of Medicine, The University of Hong Kong, Hong Kong, SAR, China

3 Department of Oral Surgery and Stomatology, School of Dental Medicine, University of Bern, Bern, Switzerland
Conclusions Most of the sinuses analyzed (79.5\%) did not present any MRC, and only $28.6 \%$ of the cysts diagnosed were found on the floor of the maxillary sinus. The mean dimension of the MRCs measured $6.28 \pm 2.93 \mathrm{~mm}$. No influencing factors on the presence or absence of MRCs were found in the present study.

Clinical relevance Most MRCs were not located on the floor of maxillary sinus. Future studies should assess their impact on surgical interventions in the sinus.

Keywords Maxillary sinus · Mucous retention cyst $\cdot$ Cone beam computed tomography

\section{Introduction}

A recent systematic review highlighted that one of the main reasons for cone beam computed tomography (CBCT) imaging in dental medicine was the assessment of the residual ridge and maxillary sinus prior to dental implant placement or sinus floor elevation (SFE) [1]. CBCT has been recommended for preoperative evaluation of the available bone in the posterior maxilla and assessing health or pathology of the maxillary sinus by several professional organizations [2-4]. The visualization of maxillary sinuses for diagnostic purposes has been also recommended in other disciplines of dental medicine such as endodontology for assessment of teeth undergoing apical surgery [5], orthodontics [6], and for the analysis of cysts or neoplasias in the maxillary region [7].

Pathologies in the maxillary sinus can be roughly classified into inflammatory, iatrogenic, traumatic, neoplastic, odontogenic, congenital, and bone-related [8]. Mucous retention cysts (MRC) were reported to be the most common manifestation due to inflammatory changes following 
localized or generalized flat thickenings of the Schneiderian membrane accounting for $10 \%$ of all sinus abnormalities [8]. MRCs are described as a homogeneous soft-tissue mass without cortical lining and appearing as a dome-shaped radiopacity extending towards the lumen of the maxillary sinus [9]. Most studies assessing MRCs were using panoramic radiographs [10-13]. There was one CT study on findings in rhinosinusitis patients in which MRCs were reported as incidental findings [14]. Similarly for CBCT imaging, MRCs were usually reported as incidental findings and not as a primary outcome variable, while generally assessing the frequency of anatomical variations or pathology in the entire maxillofacial region [15-17]. The association between MRCs and dento-alveolar pathology remains inconclusive, as some studies reported a correlation [18, 19], while others did not $[12,13,20]$. Regarding the exact locations of MRCs within the maxillary sinuses (i.e., whether they are situated predominantly at the floor, roof, or wall), there is no conclusive data available in the literature to date.

The purpose of the present case-control study was to evaluate the frequency, location, and dimension of mucous retention cysts based on an evaluation of the entire maxillary sinus using CBCT images. Furthermore, potential contributing factors to the occurrence of MRCs such as gender, age or dental pathology will be analyzed.

\section{Material and methods}

\section{Patient sample}

The present study included all CBCT scans (ProMax 3D Mid, Planmeca Oy, Helsinki, Finland) from patients referred to Oral and Maxillofacial Radiology, Applied Oral Sciences, Faculty of Dentistry, The University of Hong Kong between January 2016 and March 2017. These imaging data sets were retrospectively screened applying the following inclusion criteria: patients $\geq 18$ years old, and one/both maxillary sinuses entirely visible on the CBCT scan. The CBCT images were excluded if:

1. None of the maxillary sinuses were completely visible;

2. Surgeries (e.g., ENT, maxillofacial) had been performed or a history of trauma was known in the region of the maxillary sinuses;

3. The maxillary sinus region was not free of artifacts (acquisition or patient-related); or

4. Pathology from anterior teeth (canine-to-canine) impinging into the maxillary sinuses.

The medical history of the patients was searched to collect their demographic data of gender and age at the time of imaging. The study followed the guidelines of the declaration of
Helsinki. The study protocol was submitted to and approved by the local institutional review board (IRB) of the University of Hong Kong/Hospital Authority Hong Kong West Cluster (approval number UW 16-495).

\section{Radiographic image analysis}

CBCT images were analyzed on a Philips 223 V LED monitor with a resolution of $1920 \times 1080$ pixels (Philips, Amsterdam, Netherlands). Data were reconstructed with slices of $0.5 \mathrm{~mm}$ thickness and either a 0.2 or a $0.4 \mathrm{~mm}$ voxel size. The MRCs to be included and analyzed were selected by two experts experienced in oral and maxillofacial radiology (AY and $\mathrm{RT}$ ), and disagreements were resolved by discussion. A single examiner (AY) performed all measurements. All linear measurements were performed twice with a minimal time gap of 1 month between the two assessments to test for intra-observer reliability (repeatability). For further data analysis, the first readings were utilized.

Image analysis was performed using a dedicated imageprocessing software (Romexis Version 4.4.0.R, Planmeca Oy, Helsinki, Finland). In a first evaluation, the type and frequency of morphological changes (mucosa and bone) in the maxillary sinuses were classified. Maxillary sinus changes were recorded radiographically and coded based on criteria adapted and modified from Soikkonen and Ainamo (1995) as used in several previous studies [21-23]:

(0) Inconspicuous/no thickening of the Schneiderian membrane;

(1) Flat, shallow thickening of the Schneiderian membrane (> $4 \mathrm{~mm}$ );

(2) Semispheric thickening of the membrane (suspected MRCs);

(3) Complete opacification of the sinus;

(4) Mixed flat and semispherical thickenings; or.

(5) Other (e.g., bone destruction, cyst, aspergilloma, foreign body, suspected neoplasia).

Based on this classification, findings were grouped into (a) patients/sinuses with MRCs (test), and (b) patients/ sinuses (healthy or with any other mucosal changes) without MRCs (control). If MRCs in the form of semispheric thickenings of the Schneiderian membrane were identified, their location (roof, floor, walls of the maxillary sinus) was recorded. The widest diameter (in $\mathrm{mm}$ ) of the lesions was measured on coronal slices, and additionally on sagittal (if the lesion was located on the roof or floor of the sinus) or on axial slices (if the lesion was located on the walls of the sinus) (Fig. 1). For further analysis, a mean of both values was calculated. Only lesions wider than $3 \mathrm{~mm}$ in both dimensions were included.

For both groups (test and control), the status of the dentition from the first premolar to the second molar with regard to the maxillary sinus under investigation was 
Fig. 1 The widest diameter of a mucous retention cyst (red line) was measured from a coronal and a sagittal slice, if located on the roof/floor or $\mathbf{b}$ a coronal and an axial slice, if it was located on the walls of the maxillary sinus
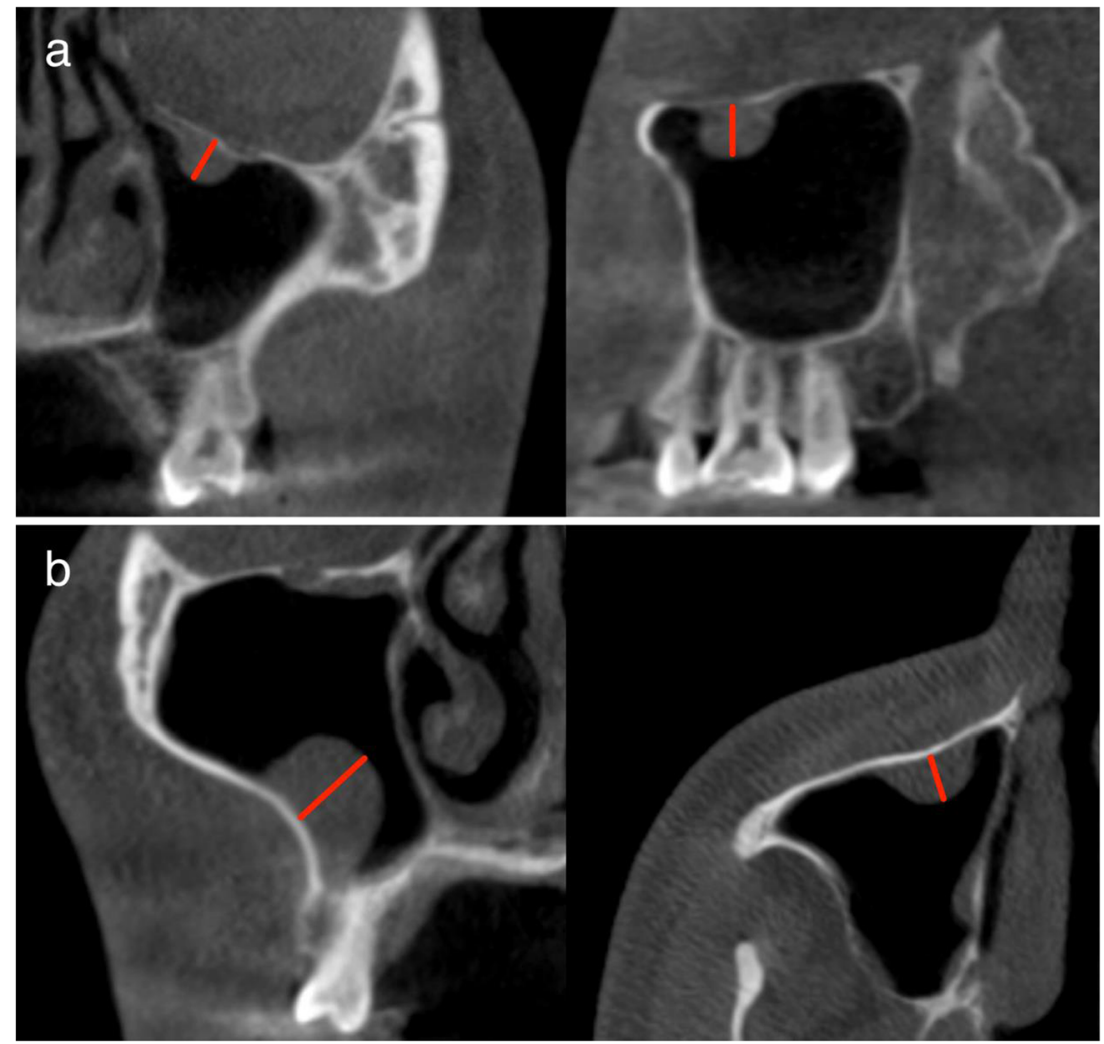

classified into: completely edentulous, partially edentulous, and dentate. If teeth were present, their status was evaluated to account for potential endodontic or periodontal pathology that could influence the status of the maxillary sinus. The endodontic status of the teeth in the respective posterior maxilla was classified into (assigning the largest code value whenever applicable):

1. No endodontic pathology or treatment;

2. Endodontic treatment(s) without visible apical pathology;

3. Apical lesion(s) with or without visible endodontic treatment(s);

Similarly, teeth with periodontal pathology were classified into (assigning the largest code value whenever applicable):

1. No periodontal lesions;

2. Horizontal and/or vertical periodontal bone lesions without furcation involvement;

3. Horizontal and/or vertical periodontal bone lesions with furcation involvement.

\section{Statistical analysis}

All data were first analyzed descriptively. For further evaluation, the assessment for differences between groups regarding age and gender was done on the patient level. The assessment for differences between groups regarding sinus location, type of dentition, endodontic status, and periodontal pathology was done on the sinus level. The significance of categorical independent variable (such as gender) was evaluated with Pearson's chi square or Fisher's exact tests on the patient level. The significance of continuous independent variable (i.e., age) was evaluated with nonparametric test (Mann-Whitney $U$ test) if the data did not follow normal distribution on the patient level. For the sinus level, to account for the possible patient clustering effect, the significance of categorical independent variables (such as side of the maxillary sinus, etc.) were evaluated with logistic regression using generalized estimating equations (GEE) model [24]. Further assessments within the MRC group were done on the cyst level. To account for the possible patient clustering effect, the significances of each independent variable on the presence in different locations (binary responses) were evaluated with separated logistic regressions using GEE models. The significances of each independent variable on the dimension (continuous response) were evaluated with GEE models with identity link function.

Intra-rater reliability was assessed using one-way random, single measures intra-class correlation coefficients (ICC). The significance level chosen for all statistical tests was $p \leq 0.05$. All analyses were performed in SPSS (Version 24.0, IBM Corp., Armonk, New York, USA). 


\section{Results}

\section{Population and imaging details}

A total of 537 CBCT scans were screened initially. After screening, 352 CBCT scans were excluded because they did not completely visualize one or both maxillary sinuses. Another 29 CBCT scans were excluded due to previous surgical interventions or pathology in the maxillary sinuses. Thus, a total of $156 \mathrm{CBCT}$ scans fulfilled the inclusion criteria (Table 1). The field of view (FOV, in $\mathrm{mm}$, diameter $\times$ height) included scans with $8 \times 8,10 \times 6,10 \times 10,20 \times 6,20 \times 10$, and $20 \times 17$. The most common FOV used were $20 \times 17(119 /$ $76.3 \%)$ and $20 \times 10(24 / 15.4 \%)$. The most frequent indications for the scans were maxillofacial surgery (41/26.3\%), followed by oral surgery including dental implant treatment planning $(21 / 13.5 \%)$.

Of these 156 scans, 124 scans $(79.5 \%)$ did not present any semispheric thickening in the visible sinuses. There were 40 maxillary sinuses (20 left, 20 right) with a total of 56 MRCs among 32 patients $(20.5 \%$; 13 males, 19 females) with a mean age of 29 (range 22 to 64 years; Table 2). Most of these 40 sinuses were associated with a fully dentate dentition (25/ $62.5 \%$ ), with no endodontic pathology or treatment (37/ $92.5 \%$ ) and no periodontal lesions (38/95\%; Table 3).

The majority of the sinuses contained one MRC ( $n=30$ / $75 \%$ ), but one quarter contained multiple cysts (Fig. 2), such as two cysts $(n=5 / 12.5 \%)$, three cysts $(n=4 / 10 \%)$, and even four cysts $(n=1 / 2.5 \%)$. For the 56 cysts (Table 4$)$, more than half $(30 / 53.6 \%)$ were located at the walls of the sinuses (Fig. 3), whereas $16(28.6 \%)$ were found on the floor (Fig. 4), and the remaining $10(17.9 \%)$ on the roof. The mean dimension of the MRCs measured $6.28 \pm 2.93 \mathrm{~mm}$.

\section{Intra-observer repeatability}

Intra-class correlation coefficients in the diameter were 0.994 for the coronal, and 0.992 for sagittal/axial slices, while intraclass correlation coefficient in the final averaged diameter was 0.997 for the $56 \mathrm{MRCs}$. These data suggest an excellent intraobserver repeatability [25].

\section{Potential influencing factors on frequency of MRCs}

There were 270 (out of $310 / 87.1 \%$ ) sinuses without a mucous retention cyst among 147 subjects (50 males, 97 females). Most (230/85.2\%) of these sinuses were healthy (Table 2), associated with a dentate dentition $(182 / 67.4 \%)$, without endodontic pathology or treatment $(254 / 94.1 \%)$, and no periodontal lesions $(258 / 95.6 \%)$. The analysis showed that gender, age, sinus side, status of dentition, endodontic status, and periodontal status did not have a significant influence on the presence of MRCs (Table 5).

Age and endodontic status exhibited a significant association with cyst location (Table 6). Older patients had a significantly lower chance for a cyst in the roof (odds ratio, $\mathrm{OR}=0.88,95 \%$ confidence interval, $\mathrm{CI}=0.78$ $1.00, p=0.045)$. Sinuses having endodontic pathology or treatment had a significantly higher chance to have a cyst at the floor of the maxillary sinus $(\mathrm{OR}=9.00,95 \%$ $\mathrm{CI}=1.56-51.85, p=0.014)$. The periodontal status exhibited a significant inverse association with cyst dimension (Table 6). Sinuses having no periodontal lesions on average had a significantly larger cyst dimension $(95 \%$ $\mathrm{CI}=0.03-1.75, p=0.043)$.

\section{Discussion}

The presence of at least one MRC in the maxillary sinus was found in 32 of the 156 CBCT scans analyzed in the present study (a total of 56 MRCs among 32 patients), which results in a frequency of $20.5 \%$ of the patients. This rate is considerably higher than those reported for Brazilian populations varying from 3.6 to $10.1 \%$, which also only assessed CBCT scans with complete visualization of one or both maxillary sinuses $[8,20]$. Similarly, a study in a Turkish population reported an occurrence rate of $2.9 \%$, but did not specify the FOV applied for sinus evaluation [15]. On the other hand, the rate found in the present study is similar to that of another Brazilian population $(21.4 \%)$ that underwent CBCT scans to visualize at least the lower third of the maxillary sinus prior to
Table 1 Total number of patients and sinuses included in the analyses
Patient level

\begin{tabular}{lllll}
\hline & $\begin{array}{l}\text { CBCT visualizing: } \\
\text { Only right maxillary } \\
\text { sinus }\end{array}$ & $\begin{array}{l}\text { Only left maxillary } \\
\text { sinus }\end{array}$ & $\begin{array}{c}\text { Both maxillary } \\
\text { sinuses }\end{array}$ & \\
Males & 0 & 0 & 53 & 53 \\
Females & 0 & 2 & 101 & 103 \\
Total & 0 & 2 & 154 & 156 \\
\hline
\end{tabular}

At the sinus level, there were 154 right maxillary sinuses, 156 left maxillary sinuses, and a total of 310 maxillary sinuses 
Table 2 Demographic data of maxillary sinuses diagnosed with or without a mucous retention cyst (MRC)

\begin{tabular}{llll}
\hline & With MRC & Without MRC & Total \\
\hline $\begin{array}{l}\text { Number of sinuses involved } \\
\text { (1) Left }\end{array}$ & $20(12.8 \%)$ & $136(87.2 \%)$ & 156 \\
(2) Right & $20(13.0 \%)$ & $134(87.0 \%)$ & 154 \\
(3) Total & $40(12.9 \%)$ & $270(87.1 \%)$ & 310 \\
Gender & & & 63 \\
(1) Male & $13(20.6 \%)$ & $50(79.4 \%)$ & 116 \\
(2) Female & $19(16.4 \%)$ & $97(83.6 \%)$ & $179^{*}$ \\
(3) Total & $32(17.9 \%)$ & $147(82.1 \%)$ & \\
Age (mean \pm SD) & $29.0 \pm 10.8$ & $31.2 \pm 14.6$ & \\
Morphological changes of sinus & & 230 & \\
(0) Healthy/shallow thickening of $<4$ mm & NA & 23 & \\
(1) Mucosal thickening of $>4$ mm & NA & 6 & \\
(3) Complete opacification of sinus & NA & 6 & \\
(4) Mixed flat/semispherical thickening & NA & 5 & \\
(5) Other (e.g.. bone destruction, foreign & NA & & \\
body, aspergilloma, suspected neoplasia) & &
\end{tabular}

Percentages of the relative risk of MRC per subcategory were shown

*There were 23 patients who had one sinus with MRC and one without, thus there were $(179-23=156$ patients $)$ implant insertion [17]. For studies using panoramic radiography, the frequency of MRCs was reported to be within the range of $5.2-14 \%[10,11,13]$. It seems that the frequency of $\mathrm{MRCs}$ reported in the literature exhibits a large variability, which might be related to the type of imaging (2- versus 3-dimensional), the FOV applied, the aspect of the maxillary sinus visualized (partial or entire), and also the ethnic background.
Several studies analyzing panoramic views have reported that all MRCs were located at the floor of the sinus $[10,11]$. In the current study, more than half $(53.6 \%)$ of the MRCs were located at the walls of the sinuses and another $17.8 \%$ were diagnosed at the roof, leaving only $28.6 \%$ located on the floor. It should be noted that a Korean study using Waters view to track the changes of MRCs $(n=40)$ reported that $70 \%$ of MRCs were attached
Table 3 Status of the dentition and dental pathology (endodontic or periodontal) associated with the maxillary sinuses diagnosed with or without a mucous retention cyst (MRC)

\begin{tabular}{|c|c|c|c|}
\hline & $\begin{array}{l}\text { With MRC } \\
(n=40)\end{array}$ & $\begin{array}{l}\text { Without MRC } \\
(n=270)\end{array}$ & $\begin{array}{l}\text { Total } \\
(n=310)\end{array}$ \\
\hline \multicolumn{4}{|l|}{ Status of dentition (from first premolar to second molar) } \\
\hline (1) Dentate & $25(12.1 \%)$ & $182(87.9 \%)$ & 207 \\
\hline (2) Partially edentulous & $15(16.7 \%)$ & $75(83.3 \%)$ & 90 \\
\hline (3) Completely edentulous & 0 & $13(100 \%)$ & 13 \\
\hline \multicolumn{4}{|l|}{ Endodontic status } \\
\hline (1) No endodontic pathology or treatment & $37(12.7 \%)$ & $254(87.3 \%)$ & 291 \\
\hline $\begin{array}{l}\text { (2) Endodontic treatment(s) without visible apical } \\
\text { pathology }\end{array}$ & $1(10 \%)$ & $9(90 \%)$ & 10 \\
\hline $\begin{array}{l}\text { (3) Apical lesion(s) with or without visible endodontic } \\
\text { treatment(s) }\end{array}$ & $2(22.2 \%)$ & $7(77.8 \%)$ & 9 \\
\hline \multicolumn{4}{|l|}{ Periodontal pathology } \\
\hline (1) No periodontal lesions & $38(12.8 \%)$ & $258(87.2 \%)$ & 296 \\
\hline $\begin{array}{l}\text { (2) Horizontal and/or vertical periodontal bone lesions } \\
\text { without furcation involvement }\end{array}$ & $2(100 \%)$ & 0 & 2 \\
\hline $\begin{array}{l}\text { (3) Horizontal and/or vertical periodontal bone lesions } \\
\text { with furcation involvement }\end{array}$ & 0 & $12(100 \%)$ & 12 \\
\hline
\end{tabular}

Percentages of the relative risk of MRC per subcategory were shown 


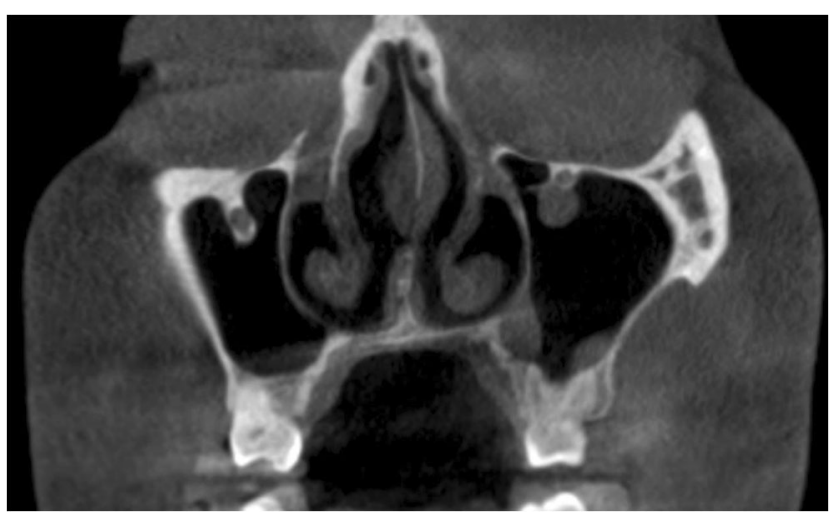

Fig. 2 Representative CBCT image of a patient with three mucous retention cysts that were diagnosed at the walls (2) and roof (1) of the left maxillary sinus (original FOV of $20 \times 17 \mathrm{~mm}$ )

to the floor, $25 \%$ were attached to the walls, and $5 \%$ were attached to the roof [26].

The MRCs in the current cohort were equally distributed in left and right sinuses, and similarly distributed between females and males. The involved patients were in their late $20 \mathrm{~s}$ on average. These findings were more or less consistent with previous reports $[8,10-12,20]$. The averaged MRC measured in the current study was approximately $6.28 \mathrm{~mm}$, which was smaller than the $11.58 \mathrm{~mm}$ reported by Nascimento and co-workers [20]. Regarding the measurements in the present study, the voxel sizes of the CBCT images were relatively large, i.e., 0.2 or $0.4 \mathrm{~mm}$. It might be that using CBCT images with a higher resolution could result in more precise or slightly different linear measurements. Other studies using either $\mathrm{CBCT}$ imaging or panoramic views did not report on the dimensions of MRCs [8, 10-12].

There was no significant association between the presence of MRCs (test versus control group) and dento-alveolar

Table 4 Demographics of mucous retention cysts

\begin{tabular}{ll}
\hline & Number of cysts \\
\hline Side & \\
(1) Left & 31 \\
(2) Right & 25 \\
Location & \\
(1) Roof & 10 \\
(2) Floor & 16 \\
(3) Walls & 30 \\
Dimensions (in mm) & \\
(1) Mean \pm SD & $6.28 \pm 2.93$ \\
(2) Median (1st quartile, 3rd quartile) & $5.59(4.08,7.59)$ \\
(3) Maximum & 16.20 \\
(4) Minimum & 3.05 \\
\hline
\end{tabular}

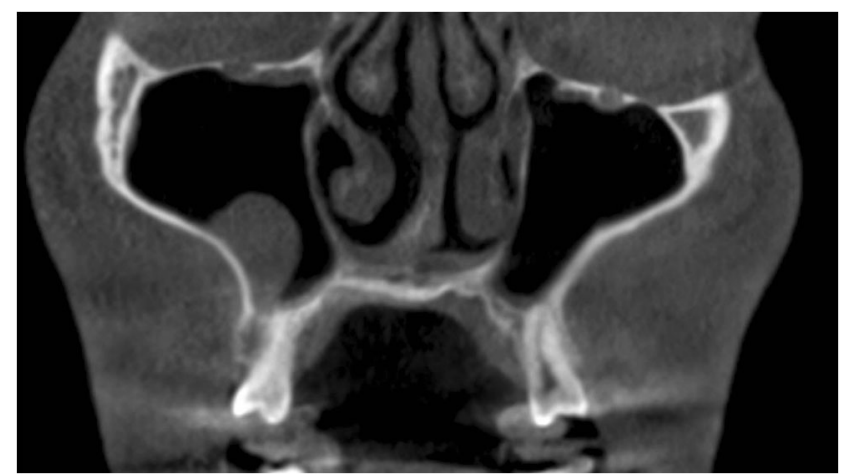

Fig. 3 CBCT image of a patient with a mucous retention cyst that was diagnosed at the lateral wall of the right maxillary sinus (original FOV of $20 \times 17 \mathrm{~mm}$ )

pathology in the present study, which was consistent with several previous reports $[10,11,20]$. In terms of location and size of MRCs, however, our statistics demonstrated that sinuses with endodontic involvement of the associated teeth had a larger chance to have MRCs on the sinus floor, and MRCs were slightly larger in sinuses without periodontal pathology in associated teeth. Nevertheless, these reported associations have to be considered with some caution, as the number of cases with endodontic or periodontal pathologies included were small, periodontal disease is not primarily assessed by CBCT imaging, and usually a small FOV is applied for patients requiring an evaluation of endodontic conditions [27]. In cases using a small FOV, only aspects of the maxillary sinuses were depicted, and the respective scans excluded from further analysis in this study. This is also reflected here, as the primary indication for 3-dimensional imaging in the current study was treatment planning of maxillofacial procedures.

The presence of an MRC is relevant to dentistry and particularly should be taken into consideration during implant

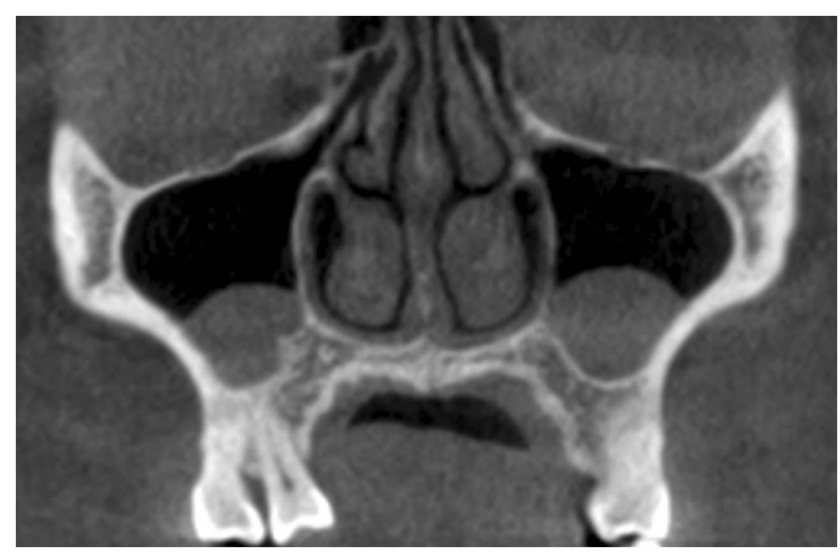

Fig. 4 CBCT image of a patient with mucous retention cysts diagnosed simultaneously at the floor of both maxillary sinuses (original FOV of $20 \times 17 \mathrm{~mm}$ ) 
Table 5 Analysis of demographic and clinical factors as influencing parameters on the presence of mucous retention cysts (test versus control group)

\begin{tabular}{lcc}
\hline Influencing factors & Test & $\begin{array}{l}p \\
\text { value }\end{array}$ \\
\hline $\begin{array}{l}\text { Patient level ( } \boldsymbol{n}=\mathbf{3 2} \text { in test group, } \boldsymbol{n}=\mathbf{1 4 7} \text { in control group) } \\
\text { Gender } \\
\text { Age }\end{array}$ & $\begin{array}{c}\text { Chi-square test } \\
\text { Mann-Whitney }\end{array}$ & 0.478 \\
test & 0.809 \\
Sinus level ( $\boldsymbol{n}=\mathbf{4 0}$ in test group, $\boldsymbol{n}=\mathbf{2 7 0}$ in control group) & \\
Location (left versus right) & GEE with logit & 0.952 \\
& link function & \\
Status of the dentition (completely & GEE with logit & NA \\
edentulous, partially edentulous & link function & \\
and dentate) & GEE with logit & 0.796 \\
Endodontic status & link function & \\
Periodontal status & GEE with logit & NA \\
& link function & \\
\hline
\end{tabular}

Entries in bold represent statistically significant difference $(p<0.05)$

$G E E$ generalized estimating equations model assuming an independence working correlation matrix, $N A$ not applicable if there existed zero frequency in the cells

treatment planning. Depending on the characteristics of the morphological changes of the Schneiderian membrane, differential diagnoses such as potential malignancy should be eliminated before implant surgery and sinus floor elevation procedures [21]. Whereas true mucoceles may expand sinus walls and erode underlying bone, MRCs do not exhibit such a destructive nature [28]. Another pathology commonly identified in the maxillary sinus are polyps, which radiographically exhibit a more irregular shape and configuration, an ability to expand the sinus cavity and extend into the nasal cavity [29]. These characteristics distinguish polyps clearly from MRCs.

According to Wang and co-workers, a "wait and see" strategy for MRCs is recommended, based on their observations that MRCs might remain unchanged or disappear totally [26]. Nevertheless, as SFE causes postoperative edema, the presence of a large sinus cyst may increase the risk of blockage of the sinus ostium, leading to fluid accumulation and sinusitis [28]. A case report was published to describe the removal of a MRC before SFE [30], where the Schneiderian membrane was intentionally perforated to remove the MRC and then was repaired with a resorbable collagen membrane. Aspiration of the liquid within the MRCs before performing sinus grafting has also been reported [31]. One group mentioned that by using preoperative panoramic radiographs for planning purposes, they aborted SFE procedures when encountering MRCs during surgery [32]. Weitz and co-workers were using unspecified preoperative imaging to diagnose sinus septa and also discontinued SFE when detecting a
Table 6 Analysis of demographic and clinical factors as influencing parameters on the location and dimension of mucous retention cysts (test group only)

\begin{tabular}{|c|c|c|}
\hline Influencing factors & Test & $p$ value \\
\hline \multicolumn{3}{|l|}{ Gender } \\
\hline \multicolumn{3}{|l|}{ 1. Location } \\
\hline Floor & GEE with logit link function & 0.759 \\
\hline Roof & GEE with logit link function & 0.932 \\
\hline Lateral walls & GEE with logit link function & 0.737 \\
\hline 2. Dimension & GEE with identity link function & 0.733 \\
\hline \multicolumn{3}{|l|}{ Age } \\
\hline \multicolumn{3}{|l|}{ 1. Location } \\
\hline Floor & GEE with logit link function & 0.194 \\
\hline Roof & GEE with logit link function & 0.045 \\
\hline Lateral walls & GEE with logit link function & 0.988 \\
\hline 2. Dimension & GEE with identity link function & 0.349 \\
\hline \multicolumn{3}{|c|}{ Status of the dentition } \\
\hline \multicolumn{3}{|l|}{ 1. Location } \\
\hline Floor & GEE with logit link function & 1.000 \\
\hline Roof & GEE with logit link function & 0.162 \\
\hline Lateral walls & GEE with logit link function & 0.359 \\
\hline 2. Dimension & GEE with identity link function & 0.915 \\
\hline \multicolumn{3}{|c|}{ Endodontic status (No endodontic pathology or treatment VS. Others) } \\
\hline \multicolumn{3}{|l|}{ 1. Location } \\
\hline Floor & GEE with logit link function & 0.014 \\
\hline Roof & GEE with logit link function & 0.596 \\
\hline Lateral walls & GEE with logit link function & NA \\
\hline 2. Dimension & GEE with identity link function & 0.400 \\
\hline \multicolumn{3}{|l|}{ Periodontal status } \\
\hline \multicolumn{3}{|l|}{ 1. Location } \\
\hline Floor & GEE with logit link function & 0.871 \\
\hline Roof & GEE with logit link function & NA \\
\hline Lateral walls & GEE with logit link function & 0.688 \\
\hline 2. Dimension & GEE with identity link function & 0.043 \\
\hline
\end{tabular}

Entries in bold represent statistically significant difference $(p<0.05)$

GEE generalized estimating equations model assuming an independence working correlation matrix, NA not applicable if there existed zero frequency in the cells

MRC [33]. The variability to manage MRCs mentioned above is showing that there has been no clear recommendation to date for management of MRCs prior and during implant surgery. As our current study has demonstrated that not even one third of all MRCs were located on the floor of the maxillary sinus, future studies should address the potential causes of MRCs and evaluate the long-term behavior of these lesions. This might also help to know, if their presence is detrimental to surgeries related to SFE.

Acknowledgements The authors are grateful to Ms. Kar Yan Li, Centralized Research Lab, Faculty of Dentistry, The University of Hong Kong, for her valuable assistance regarding the statistical analysis. 
Funding This study has been funded by departmental funds only. No external funding has been received.

\section{Compliance with ethical standards}

Conflict of interest The authors declare that they have no competing interests.

Ethical approval All procedures performed were in accordance with the ethical standards of the institutional and/or national research committee and with the 1964 Helsinki declaration and its later amendments or comparable ethical standards. The study protocol was submitted to and approved by the local institutional review board (IRB) of the University of Hong Kong / Hospital Authority Hong Kong West Cluster (approval number UW 16-495).

Informed consent For this type of study (retrospective study), formal consent is not required.

\section{References}

1. Vogiatzi T, Kloukos D, Scarfe WC, Bornstein MM (2014) Incidence of anatomical variations and disease of the maxillary sinuses as identified by cone beam computed tomography: a systematic review. Int J Oral Maxillofac Implants 29: 1301-1314

2. Benavides E, Rios HF, Ganz SD, An C-H, Resnik R, Reardon GT, Feldman SJ, Mah JK, Hatcher D, Kim M-J (2012) Use of cone beam computed tomography in implant dentistry: the International Congress of Oral Implantologists consensus report. Implant Dent 21:78-86

3. Bornstein MM, Scarfe WC, Vaughn VM, Jacobs R (2014) Cone beam computed tomography in implant dentistry: a systematic review focusing on guidelines, indications, and radiation dose risks. Int J Oral Maxillofac Implants 29:55-77

4. Harris D, Horner K, Gröndahl K, Jacobs R, Helmrot E, Benic GI, Bornstein MM, Dawood A, Quirynen M (2012) EAO guidelines for the use of diagnostic imaging in implant dentistry 2011. A consensus workshop organized by the European Association for Osseointegration at the Medical University of Warsaw. Clin Oral Implants Res 23:1243-1253

5. Rigolone M, Pasqualini D, Bianchi L, Berutti E, Bianchi SD (2003) Vestibular surgical access to the palatine root of the superior first molar: "low-dose cone-beam" CT analysis of the pathway and its anatomic variations. J Endod 29:773-775

6. Kusnoto B, Kaur P, Salem A, Zhang Z, Galang-Boquiren MT, Viana G, Evans CA, Manasse R, Monahan R, BeGole E (2015) Implementation of ultra-low-dose CBCT for routine 2D orthodontic diagnostic radiographs: cephalometric landmark identification and image quality assessment. Semin Orthod 21:233-247

7. Chindasombatjaroen J, Poomsawat S, Klongnoi B (2012) Calcifying cystic odontogenic tumor associated with other lesions: case report with cone-beam computed tomography findings. Oral Surg Oral Med Oral Pathol Oral Radiol 113:414-420

8. Rege ICC, Sousa TO, Leles CR, Mendonça EF (2012) Occurrence of maxillary sinus abnormalities detected by cone beam CT in asymptomatic patients. BMC Oral Health 12:30

9. Gardner DG (1984) Pseudocysts and retention cysts of the maxillary sinus. Oral Surg Oral Med Oral Pathol 58:561-567

10. MacDonald-Jankowski D (1993) Mucosal antral cysts in a Chinese population. Dentomaxillofac Radiol 22:208-210
11. MacDonald-Jankowski D (1994) Mucosal antral cysts observed within a London inner-city population. Clin Radiol 49:195-198

12. Rodrigues C, Freire G, Silva L, da Silveira MF, Estrela C (2014) Prevalence and risk factors of mucous retention cysts in a Brazilian population. Dentomaxillofac Radiol 38:480-483

13. Vallo J, Suominen-Taipale L, Huumonen S, Soikkonen K, Norblad A (2010) Prevalence of mucosal abnormalities of the maxillary sinus and their relationship to dental disease in panoramic radiography: results from the health 2000 health examination survey. Oral Surg Oral Med Oral Pathol Oral Radiol Endod 109:e80-e87

14. Jones N, Strobl A, Holland I (1997) A study of the CT findings in 100 patients with rhinosinusitis and 100 controls. Clin Otolaryngol Allied Sci 22:47-51

15. Çaglayan F, Tozoglu Ü (2012) Incidental findings in the maxillofacial region detected by cone beam CT. Diagn Intery Radiol 18:159-163

16. Kang B-C, Yoon S-J, Lee J-S, Al-Rawi W, Palomo JM (2011) The use of cone beam computed tomography for the evaluation of pathology, developmental anomalies and traumatic injuries relevant to orthodontics. Semin Orthod 17:20-33

17. Pelinsari Lana J, Moura Rodrigues Carneiro P, de Carvalho MV, Eduardo Alencar de Souza P, Ricardo Manzi F, Campolina Rebello Horta M (2012) Anatomic variations and lesions of the maxillary sinus detected in cone beam computed tomography for dental implants. Clin Oral Implants Res 23: 1398-1403

18. Hauman C, Chandler N, Tong D (2002) Endodontic implications of the maxillary sinus: a review. Int Endod J 35:127-141

19. Moskow BS (1992) A histomorphologic study of the effects of periodontal inflammation on the maxillary sinus mucosa. J Periodontol 63:674-681

20. Nascimento EHL, Pontual MLA, Pontual AA, Freitas DQ, Perez DEC, Ramos-Perez FM (2016) Association between odontogenic conditions and maxillary sinus disease: a study using cone-beam computed tomography. J Endod 42:1509-1515

21. Bornstein MM, Horner K, Jacobs R (2017) Use of cone beam computed tomography in implant dentistry: current concepts, indications and limitations for clinical practice and research. Periodontol 2000 73:51-72

22. Schneider AC, Brägger U, Sendi P, Caversaccio MD, Buser D, Bornstein MM (2013) Characteristics and dimensions of the sinus membrane in patients referred for single-implant treatment in the posterior maxilla: a cone beam computed tomographic analysis. Int $\mathrm{J}$ Oral Maxillofac Implants 28: 587-596

23. Soikkonen K, Ainamo A (1995) Radiographic maxillary sinus findings in the elderly. Oral Surg Oral Med Oral Pathol Oral Radiol Endod 80:487-491

24. Mancl LA, Leroux B, DeRouen T (2000) Between-subject and within-subject statistical information in dental research. J Dent Res 79:1778-1781

25. Fleiss JL, Levin B, Paik MC (2013) Statistical methods for rates and proportions, 3rd edn. John Wiley \& Sons, New Jersey

26. Wang JH, Jang YJ, Lee BJ (2007) Natural course of retention cysts of the maxillary sinus: long-term follow-up results. Laryngoscope 117:341-344

27. Patel S, Durack C, Abella F, Roig M, Shemesh H, Lambrechts P, Lemberg K (2014) European Society of Endodontology position statement: the use of CBCT in endodontics. Int Endod J 47:502-504

28. Mardinger O, Manor I, Mijiritsky E, Hirshberg A (2007) Maxillary sinus augmentation in the presence of antral pseudocyst: a clinical approach. Oral Surg Oral Med Oral Pathol Oral Radiol Endod 103:180-184 
29. Towbin R, Dunbar J, Bove K (1979) Antrochoanal polyps. AJR Am J Roentgenol 132:27-31

30. Pikos MA (2008) Maxillary sinus membrane repair: update on technique for large and complete perforations. Implant Dent 17:24-31

31. Maiorana $\mathrm{C}$, Beretta $\mathrm{M}$, Benigni $\mathrm{M}$, Cicciù $\mathrm{M}$, Stoffella $\mathrm{E}$, Grossi G (2012) Sinus lift procedure in presence of mucosal cyst: a clinical prospective study. JIACD 4:54-60
32. Becker ST, Terheyden H, Steinriede A, Behrens E, Springer I, Wiltfang J (2008) Prospective observation of 41 perforations of the Schneiderian membrane during sinus floor elevation. Clin Oral Implants Res 19:1285-1289

33. Weitz DS, Geminiani A, Papadimitriou DE, Ercoli C, Caton JG (2014) The incidence of membrane perforation during sinus floor elevation using sonic instruments: a series of 40 cases. Int J Periodontics Restor Dent 34:105-112 